\title{
Increasing Performance of SDR-based Collision-Free RFID Systems
}

\author{
${ }^{1}$ Danilo De Donno, ${ }^{2}$ Vasileios Lakafosis, ${ }^{1}$ Luciano Tarricone, and ${ }^{2}$ Manos M. Tentzeris \\ ${ }^{1}$ Innovation Engineering Department, University of Salento, Lecce, Italy \\ ${ }^{2}$ Department of Electrical and Computer Engineering, Georgia Institute of Technology, USA
}

\begin{abstract}
Maximizing the identification speed of Radio Frequency Identification (RFID) tagged items is one of the main challenges in the area than can bring significant benefits to a vast array of applications. The dominating UHF EPC Gen2 protocol only specifies collision avoidance algorithms and, although collision recovery techniques have been investigated by researchers in the past, no attempt has been made to leverage the application of such techniques with minor modifications to the RFID protocol and, hence, realistically improve its performance. In this paper, we show that a significant reduction of $26 \%$ in the inventory time with collision recovery is feasible in real time relying, for the first time, on actual measurements taken with a software-defined RFID Reader and off-the-shelf programmable Tags. Among the numerous applications that can benefit from an increased reliable RFID reading rate, we are highlighting the advantages of deploying our working prototype testbed in moving conveyor belt systems. In brief, we achieve a $26 \%$ belt speed increase maintaining the exact same reading reliability and up to $84 \%$ of RFID Reader power consumption savings.
\end{abstract}

Index Terms - RFID, Software-Defined Radio, tag collision, collision recovery, inventory time, power consumption.

\section{INTRODUCTION}

One of the main challenges for any Radio Frequency Identification (RFID) based application is to maximize the identification speed of tagged products. Such a speed requirement is further exacerbated in supply chain management and logistics applications, where goods and assets tracking is performed by conveyor belt systems. In this case, the undesired collision events between concurrently transmitting Tags strongly affect the time needed to successfully identify tagged items. The EPC Class-1 Generation-2 ("EPC Gen2" for short hereafter) protocol [1] for passive UHF RFID systems does not effectively address the problem. Collision avoidance algorithms are specified in the standard. However, a conventional RFID Reader possesses no capabilities of extracting useful information from the collided RFID signals of simultaneous Tag transmissions. It simply discards this exploitable data and requires a retransmission of the Tag packets, thus incurring identification delays.

Multi-packet reception has been a widely investigated topic in the literature and the feasibility of RFID Tag collision recovery has been demonstrated with real measurements. Nonetheless, to the best of our knowledge, no extra steps have ever been taken to leverage the coupling of the collision recovery techniques with minor modifications to the RFID protocol to realistically improve its performance.
In this paper, the reduction of the RFID identification time with collision recovery is analyzed, for the first time, in real time with actual measurements exploiting the functionality provided by a software-defined RFID Reader and off-theshelf programmable Tags. Essentially, the RFID performance benefits stem from very simple, yet effective changes that we propose in the way successful Tag acknowledgments from recovered collided packets are handled. The implemented changes to the RFID protocol are tested with real-time experiments and their benefits for real-world applications, including the increase of conveyor belt speeds and Reader power savings, are investigated.

\section{SDR-BASED COLLISION-AWARE RFID}

The prototyping of RFID protocols is a very challenging task since it requires flexible and programmable platforms for both ends involved in the communication, namely the Tag and the Reader. Following a hardware / software co-design approach, we rely, in this work, on the Intel Wireless Identification and Sensing Platform (WISP) Tag developed by Intel Research Seattle [2]. WISP can be powered and read by off-the-shelf UHF RFID Readers and has an on-board microcontroller for sensing and computing functions. The typical handshake mechanism with the Reader is not supported by the firmware version that comes preloaded with the WISPs, therefore we implemented it along with a simple and computationally inexpensive pseudorandom number generator for time slot selection.

On the Reader side, we exploit a Software Defined Radio (SDR) platform [3] based on the low-cost Universal Software Radio Peripheral (USRP) and the open-source GNU Radio toolkit. The conventional EPC Gen2 RFID protocol has been modified and a novel collision-aware mechanism to acknowledge multiple Tags has been implemented. In fact, although a typical RFID Reader may only communicate with at most one Tag at a time based on current single-Tag detection techniques (see Fig.1a), it has been demonstrated that multi-packet reception is practically feasible with various novel ad-hoc algorithms [4], [5]. Hence, it is realistic to assume that the Reader can separate and decode collided Tag signals. The question, however, is how can the Reader use the information extracted from the waveform separation.

In this paper, we refer to the simple collision case where two Tags transmit during the same time slot and implement the possibility for the Reader to acknowledge both Tags by sending a new command, referred to as Long $A C K(L-A C K)$ 


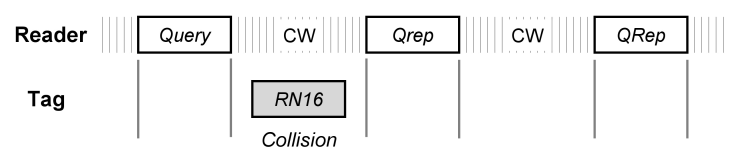

(a)

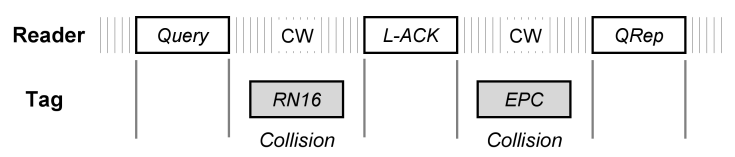

(b)

Fig. 1. RFID handshake when a (a) conventional or (b) collisionrecovery capable Reader is considered.

(ref. Fig. 1b). It should be clarified that scenarios involving collisions of more than two Tags are not examined with our experiment setup because of the lack of technically feasible collision recovery techniques that are directly portable to commercial Readers and can operate in real time. Moreover, two-Tags collisions are the most probable events in real scenarios - three times more probable than collisions involving more than two Tags for a population of 100 Tags and 100 allocated time slots. It is clear from Fig. 1 that originally a Tag collision occurs on the RN16 packet containing a 16-bit random number chosen by the Tag. Since the purpose of this work is not to propose a new technique for separating and decoding collided Tags but, instead, to highlight the benefits of such a collision recovery, the transmitted RN16 numbers will be assumed known at the Reader and, thus, are preprogrammed on the WISPs. This does not cause any loss of generality since it is reliable to expect well-consolidated and effective multi-packet reception techniques to be soon implemented. The described approach gives also rise to a collision on the $E P C$ message (ref. Fig. 1b) because both Tags will reply to the $L-A C K$. However, such a collision can be recovered with the same technique adopted for the RN16 packet. The performance gains achieved by an RFID system exploiting the proposed protocol tweaking are experimentally demonstrated in the next section.

\section{EXPERIMENTAL RESULTS}

In order to evaluate the performance gains achieved by the previously described technique involving collision-aware multi-Tag acknowledgments, we run a series of experiments in a real Tags-Reader communication scenario. We fix two WISP Tags on a polystirene sheet at a distance of 1.5 meters from the SDR Reader antennas - note that the USRP-based Reader uses a bistatic configuration, i.e. one antenna is for transmission and one for reception. Tags and antennas are mounted on easels 1.5 meters above the ground.

We instruct the Reader to perform 100 inventory cycles with 5 rounds each. If a Tag is not successfully read by the Reader during a round (for instance, because of a collision event), it will remain active in the next round. Conversely, a Tag successfully read during a round will be inactive in the

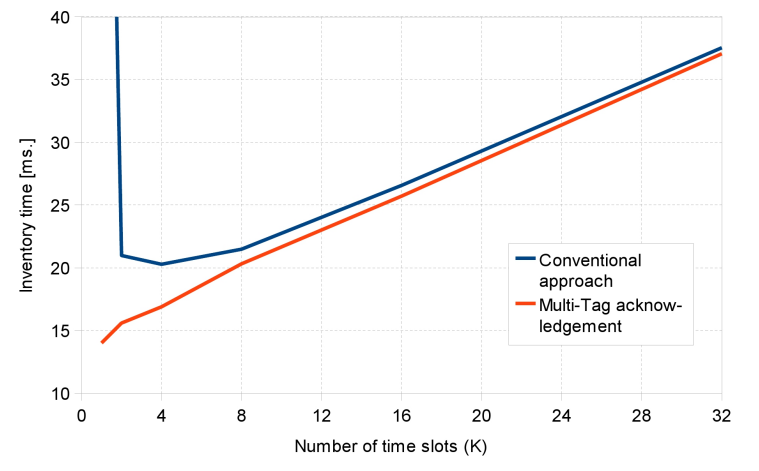

Fig. 2. Average inventory time measured experimentally at the RFID Reader under a real EPC Gen2 communication scenario.

next rounds. In order to minimize the inventory time, we force the Reader to stop an inventory cycle and pass to the next once all Tags has been read. In other words, we assume that the Reader has acquired an accurate estimate of the Tag population - such information can be inferred by well-known algorithms proposed in literature [6]. We repeat the experiments and average out the Reader logs for five different frequencies in the U.S. UHF RFID band and for five different seeds of the pseudo-random number generator implemented on the WISP Tags for the slot selection.

The following experiment demonstrates how the inventory time, i.e. the time needed for the Reader to read the whole Tag population, can be significantly reduced by the proposed modification to the RFID protocol. We assume a fixed number of $K$ time slots allocated in each of the five inventory rounds that make up an inventory cycle. Figure 2 shows the average inventory time measured at the Reader for the conventional approach, according to which collided Tag packets are discarded, and the proposed multi-Tag acknowledgment scheme, in which, instead, collided Tag packets are assumed to be recovered. It can be seen that $26 \%$ of inventory time reduction is achieved when the round consists of less than 8 time slots. Considering more than 8 allocated slots in this scenario is pointless because collision events are unlikely.

\section{APPLICATIONS}

RFID technology is widely used in supply chain management and logistics applications as a means of tracking goods and assets. A major component in large factories and warehouses for the automated identification of RFID Tags is the conveyor belt system. With daily arrivals and departures of products in the order of thousands, increasing the conveyor belt speeds without any sacrifice in the Tag identification reliability is highly desirable from a business perspective [7-9]. In this Section we briefly discuss how the EPC Gen2 enhanced performance, achieved with our proposed system and shown in the previous Section, can be utilized toward increasing the speed of the conveyor belts, which is directly 


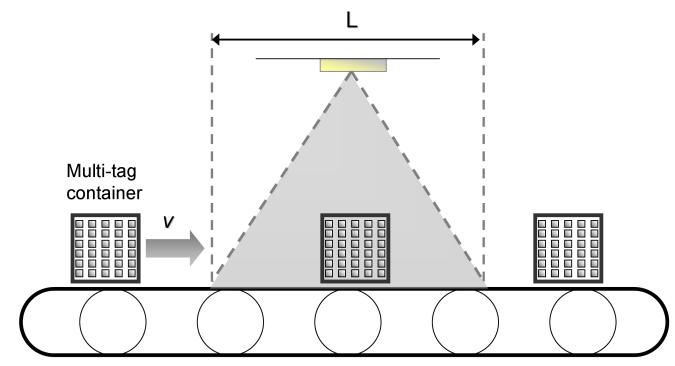

Fig. 3. Conveyor belt system for RFID-tagged items.

coupled to the increased service rate as a result of the increased arrival rate of the goods, as well as toward attaining power savings involved with the logistics operations.

Figure 3 illustrates the most common conveyor belt system [7], in which the RFID Reader's antenna "RF illuminates" vertically from above an area of length $L$ across the moving conveyor belt as the tagged products pass through the RFID "gate". The greyed triangle area corresponds to the RFID interrogation zone. Based on previous literature [10] and manufacturer data sheets [11], the size of $L$ typically ranges from $0.3 \mathrm{~m}$ to $1.5 \mathrm{~m}$. The potential of our proposed system, shown in the previous Section, is harnessed by acknowledging multiple collided packets that have been recovered, and, hence, multiple Tags are assumed to exist within the interrogation zone of the RFID reader; this is totally realistic and desired if one considers boxes containing a lot of tagged products placed on the conveyor belt.

Based on the findings in Section III, the same number of Tags needs to remain less time within the area of length $L$ to be identified successfully, compared to the current state-ofthe-art RFID systems. Specifically, as experimentally demonstrated, that is $26 \%$ less time for a population of $N=2$ Tags within the RFID Reader's interrogation zone. Simulated time reductions in the range of 8 to $17 \%$ are also reported in [5] for a population of up to $N=400$ Tags. In addition, Doppler effects can be neglected since the relative speed of the tagged objects to the Reader is low. Indicatively, even with a Tag speed of $10 \mathrm{~m} / \mathrm{s}$ the experienced Doppler shift is around 30 $\mathrm{Hz}$, which is definitely negligible if compared to the $\mathrm{KHz}-$ order of frequency shift allowed by the standard.

For the same length $L$ of the interrogation zone, the speed of the conveyor belt can be increased by the same percentage ( $26 \%$ for $N=2$ ) as the decrease of the minimum time required for a Tag population within the read range to be successfully read. That means not only increased rate of service but also lower labor cost as the factory or business operations can be completed in a shorter time.

Energy-wise, the shorter read time for the same amount of products to be identified means that the power consumption of the conveyor belt's RFID system is reduced. As a simple proof of concept, we have compared the power consumption of a commercial RFID reader, as well as that of our SDR
Reader between different operation states. It comes as no surprise that, for a typical single-antenna commercial Reader [12], the active power is $84 \%$ higher in the "Transmit /Receive" state $\left(26.3 \mathrm{~W}\right.$ for $\left.P_{T x}=0.4 \mathrm{~W}\right)$ than in its idle state $(14.3 \mathrm{~W})$, when it has no Tags to read. Similarly, the twoantenna SDR Reader used in this work consumes 37\% more power in the "Transmit/Receive" state $\left(20.3 \mathrm{~W}\right.$ for $\left.P_{T x}=0.4 \mathrm{~W}\right)$ than in the idle state $(14.8 \mathrm{~W})$. These values reflect the significant power savings achievable by our proposed system.

\section{CONCLUSION}

This work experimentally demonstrates that performance of current RFID systems can be considerably enhanced when Tag collision recovery is exploited. A flexible testbed set up with an SDR Reader and off-the-shelf programmable Tags has been used to implement a multi-Tag acknowledgment technique that achieves $26 \%$ inventory time reduction. Among the advantages that such an improved RFID system can bring in real-world applications, we have highlighted the speed increase in conveyor belts and significant gains in power consumption. In the progress of this work, we plan to further enhance the multi-Tag acknowledgment technique by exploiting beamforming with an antenna array.

\section{REFERENCES}

[1] EPCglobal, "EPC radio-frequency identify protocols - Class-1 Generation-2 UHF RFID protocol for communications at 860 MHz-960 MHz", version 1.2.0, 2008.

[2] J. R. Smith, et al.., "A Wirelessly-Powered Platform for Sensing and Computation," in Proc. of Ubicomp, pp. 495-506, 2006.

[3] M. Buettner and D. Wetherall, "A Software Radio-Based UHF RFID Reader for PHY/MAC Experimentation," in Proceedings of 2011 IEEE International Conference on RFID, April 2011.

[4] C. Angerer, R. Langwieser, and M. Rupp, "RFID Reader Receivers for Physical Layer Collision Recovery," IEEE Trans. on Communications, vol. 58, no. 12, pp. 3526-3537, Dec. 2010.

[5] J. Kimionis, et al., "Inventory Time Reduction in Gen2 with Single-Antenna Separation of FM0 RFID Signals," in Proc. of 2011 IEEE International Conference on RFID, April 2011.

[6] H. Vogt, "Efficient Object Identification with Passive RFID Tags," PERVASIVE 2002 - LNCS, vol. 2414, 2002.

[7] X. Lei, et al., "Efficient Tag Identification in Mobile RFID Systems," in Proc. of IEEE INFOCOM 2010, March 2010.

[8] L. Simon, P. Saengudomlert, and U. Ketprom, "Speed Adjustment Algorithm for an RFID Reader and Conveyor Belt System Performing Dynamic Framed Slotted Aloha," in Proc. of 2008 IEEE International Conference on RFID, April 2008.

[9] J. Singh, E. Olsen, K. Vorst, and K. Tripp, "RFID Tag Readability Issues with Palletized Loads of Consumer Goods," Packaging Technology and Science, 2009.

[10] C. R. Medeiros, J. R. Costa, and C. A. Fernandes, "RFID Reader Antennas for Tag Detection in Self-Confined Volumes at UHF," IEEE Antennas and Propagation Magazione, vol. 53, no. 2, pp. 39-50, April 2011.

[11] Barco RFID Products, Online: http://www.barco.cz/en/.

[12] Impinj Speedway RFID Reader, Online: www.impinj.com/. 